\title{
Dispersive Micro-Solid Phase Extraction for Sensitive Determination of Methotrexate from Human Saliva Followed by Spectrophotometric Method
}

\author{
Fatemeh Soghra Jahed $^{1,2}$, Samin Hamidi ${ }^{1 *}$, Mohammad Galehassadi
}

\begin{abstract}
For biological assessing of hospital personnel occupationally exposed to antineoplastic drugs, highly sensitive and accurate methods are required. Methotrexate (MTX) is an anticancer agent that is widely used in a variety of human cancers. For the first time, dispersive-micro solid phase extraction (D- $\mu$-SPE) has been applied for determination of low levels of MTX in saliva samples. The method is based on rapid extraction of MTX using graphene oxide adsorbent. The sample preparation time is decreased by the fact that the adsorbent dispersed in the sample solution and extraction equilibrium can be reached very fast. This significant feature which obtained with this method is of key interest for routine trace laboratory analysis. The influence of different variables on D- $\mu$-SPE was investigated. Under optimum conditions, the calibration graph was linear over the range of $10-1,000 \mathrm{ng} / \mathrm{ml}$. The relative standard deviations are better than $9.0 \%$. The proposed method was successfully applied for the determination of MTX in patient samples.
\end{abstract}

Keywords: Methotrexate- saliva- dispersive micro solid phase extraction- spectrophotometry

Asian Pac J Cancer Prev, 21 (6), 1531-1538

\section{Introduction}

Methotrexate (Figure 1) is an anticancer drug belonging to a class of chemotherapy drugs. It has the potential to be used as a treatment for cancers including breast cancer, lung cancer, leukemia, lymphoma, osteosarcoma and also used in the treatment of some autoimmune diseases. MTX mechanism of action in cancer therapy is performed by inhibiting cellular enzymes and preventing cell replication. It is generally administered orally and after absorption, most of the drug is excreted intact in urine.

Determination of MTX in biological samples is needed for therapeutic drug monitoring (TDM), pharmacokinetic studies, bioequivalence tests, toxicology, and forensic science (Fleisher, 1993; Flores et al., 2005). In cancer therapy, high-dose of MTX is used that have serious toxic effects. MTX has many serious adverse effects, such as myelosuppression. To minimize toxicity, safe and effective MTX therapy, monitoring of biological MTX level is very important to improve the safety of medicinal therapy (Emara et al., 1998; Li et al., 2015; Asadian et al., 2017). Several methods have been reported to determine the MTX in different biological matrices using high-performance liquid chromatography (HPLC) (Emara et al., 1998; Hroch et al., 2009; Moghbel et al., 2010; Uchiyama et al., 2012; Begas et al., 2013; Li et al., 2015), electrochemical impedance spectroscopy and cyclic voltammetry (Wei et al., 2014; Asadian et al., 2017), fluorescence (Nelson et al., 1977; Chen and Zhang, 2008; Jouyban et al., 2011), surface-enhanced Raman scattering (SERS) (Yang et al., 2014) and ultra performance liquid chromatography-electrospray ionization-tandem mass spectrometry (UPLC-ESI-MS/MS) analysis (Fabrizi et al., 2016). In the recent years less common or unconventional matrices have emerging more significant owing to their invasiveness over the conventional specimens for example when taking blood from children and neonates is difficult (Gallardo and Queiroz, 2008; Hamidi et al., 2016; Hamidi and Alipour-Ghorbani, 2017; Hamidi et al., 2017a; Hamidi et al., 2017b; Jouyban et al., 2017). Effectiveness of saliva was assessed as a non-invasive and alternative specimen to urine and blood in terms of ease of collection, matrix simplicity, and drug detection periods. Most of the analytical methods reported for MTX are tedious, time-consuming and need highly skilled operators. UV-Vis spectroscopy is one of the inexpensive, low cost and simplest analytical tools that routinely used in almost all of the biomedical laboratories (Ghasemi and Niazi, 2005; Samari et al., 2010; Hamidi, 2018). The most frequent detection system employed for the determination of MTX is UV-Vis (Rubino, 2001). MTX is a strongly UV-Vis absorbing substance, due to the presence of the heteroaromatic chromophore. To overcome the difficulty of poor sensitivity in UV detectors, pre-concentration 
methods have been recommended; the most important step in the determination of an analyte is sample preparation (Vas and Vekey, 2004; Hamidi and Jouyban, 2015a; Hamidi and Jouyban, 2015b; Hamidi et al., 2015).

Biological matrices more or less contain proteins, salts, and lipids which need to be treated prior to being coupled with the majority of analytical devices. A bioanalytical method has two major steps: sample preparation and sample quantification. Sample preparation is a crucial step and aims to simplify matrix and/or enrich analyte. Some estimates show that over $80 \%$ of an analysis process is dedicated to sample pre-treatment in order to introduce matrix-free extract into a measurement device. The ultimate aim of an extraction method is to provide the target analyte in solution with an appropriate concentration for quantification and to let it free from interferences that might exist in the original source of sample. Liquid-liquid extraction (LLE) and solid phase extraction (SPE) are the most popular sample preparation processes. Generally, LLE is performed with water-immiscible solvents to recover the analyte from the aqueous solution. However, LLE technique should be performed under conditions which provide a distinct and clean separation boundary between the extraction phase and the sample solution. Formation of rather stable emulsions is often a formidable obstacle during the phase separation, threatening efficient extraction performance, particularly when dealing with biological samples (Ruiz-Gutiérrez and Pérez-Camino, 2000; Poole, 2003).

Nowadays SPE is known as a general technique in sample pre-treatment. In conventional SPE, solid particles are embedded on a solid phase such as cartridge, fiber, and disk. The sample passed through the column by applying external pressure and they are usually recommended for single use (Ashri and Abdel-Rehim, 2011; Hamidi et al., 2018; Jafari and Hamidi, 2018). A sufficiently low flow-rate through the column of a specific sorbent is of prime importance in SPE. Residual solvent and sample molecules removal from the sorbents is very important in order to improve the reliability of the procedure. Another limitation of suffering in conventional SPE, in particular when dealing with complex matrices is clogging the sorbent pores which, in turn, affect the analyte retention time and sorbent capacity. Dispersive micro solid phase extraction (D- $\mu$-SPE) is a new, rapid, simple and effective type of SPE, used for pre-concentration of trace amounts of target analytes (Jouyban and Hamidi, 2017). In this technique, adsorbent was added into the sample solution and separation process achieved only by centrifugation or an external magnetic field (Jouyban and Hamidi, 2017; Khezeli and Daneshfar, 2017). Graphene and Graphene-based materials are good candidates for sample preparation as in many studies have been directly used as an adsorbent in the D- $\mu$-SPE process due to their unique properties e.g., having a high surface area to weight (2630 m2g-1), thermal and chemical stability and simple synthesis process (Ye and Shi, 2015). Graphene oxide (GO) is a functionalized form of graphene with different epoxy, hydroxyl, carbonyl and carboxyl surface groups (Ayazi, 2017). It is easily prepared from natural graphite by Hummer's method (Shahriary and Athawale,
2014). GO with polar surface functional groups and delocalized $\pi$-electron system is an appropriate adsorbent for extraction of polar and aromatic organic compounds.

In this work, GO was synthesized by the simple Hummer's method and used for extraction of MTX from real human saliva samples. UV spectroscopy was used for determination of the analyte concentration. The developed method was validated according to FDA guidance and applied for the determination of MTX levels in two patients with acute lymphoblastic leukemia.

\section{Materials and Methods}

\section{Experimental}

Materials and instrument

MTX powder was purchased from Sigma (Sigma, USA). Acetonitrile (ACN), methanol, ethanol and acetone were obtained from Scharlau (Barcelona, Spain). Sodium hydroxide $(\mathrm{NaOH})$, expanded graphite powder, sulforic acid (H2SO4, $98 \%$ ), hydrogen peroxide (H2O2, $30 \%$ ), $\mathrm{KMnO} 4$, hydrochloric acid $(\mathrm{HCl})$ were purchased from Merck (Darmstadt, Germany).

The UV-Vis spectrophotometer (Thermo, USA) accompanied with Thermo Insight software was used to determine the concentration of MTX. A $350 \mu \mathrm{l}$ quartz cell was used to record spectrums and absorbance measurements. The absorbance was recorded at the wavelength of $375 \mathrm{~nm}$.

\section{Standard solutions and saliva samples}

Stock standard solution of MTX $(1,000 \mu \mathrm{g} / \mathrm{ml})$ was constructed in methanol and stored in $-4^{\circ} \mathrm{C}$. Drug-free saliva samples were provided by volunteer donors and transferred in polypropylene microtubes and frozen. Saliva samples thawed at room temperature unassisted before daily experiments. Daily standard MTX solutions were prepared in drug-free saliva sample and diluted with the same matrix. The required calibrator solutions $(0.01,0.05$, $0.1,0.5$ and $1 \mu \mathrm{g} / \mathrm{ml})$ were prepared in the same manner. Two samples were obtained from patients receiving MTX who had signed consent forms approved by the ethics committee, Tabriz University of Medical Sciences. These Samples were also collected in polypropylene tubes and stored in refrigerator until analysis.

\section{Synthesis of $G O$}

GO was prepared and characterized by the method mentioned in our previously work (Jouyban and Hamidi, 2017). In brief, graphite (2 g) and sulfuric acid (50 ml) was purred in $1,000 \mathrm{ml}$ flask and stirred at $0-5^{\circ} \mathrm{C}$ in the ice bath for $2 \mathrm{~h}$, then potassium permanganate $(6 \mathrm{~g})$ slowly added to the mixture. In this step the reaction temperature should be controlled and keep lower than $15^{\circ} \mathrm{C}$. After that, the reaction mixture stirred at $35^{\circ} \mathrm{C}$ until pasty brownish color obtained and continuously stirred at this temperature. After $30 \mathrm{~min}$ the mixture diluted by addition of $100 \mathrm{ml}$ deionized water, the temperature rapidly reached to $98^{\circ} \mathrm{C}$ and color changed to brown. Dilution was continued by adding $200 \mathrm{ml}$ deionized water. The reaction terminates by adding $10 \mathrm{ml} \mathrm{H}_{2} \mathrm{O}_{2}$ and yellow color was appeared. After filtration, the obtained GO washed several times with 
$10 \% \mathrm{HCl}$ and deionized water and dried at $70^{\circ} \mathrm{C}$ for $24 \mathrm{~h}$.

Dispersive micro-solid phase extraction (D- $\mu-S P E)$ procedure

The D- $\mu$-SPE performance was done as follows: First $0.5 \mathrm{ml}$ of the saliva sample was put in a $15 \mathrm{ml}$ tube spiked with MTX and diluted six times with deionized water. Then, the $\mathrm{pH}$ of this solution adjusted to 4 with $30 \mu \mathrm{l}$ of $\mathrm{HCl} 0.1 \mathrm{M}$. Next, $3 \mathrm{mg} \mathrm{GO}$ weighed and dispersed into the mixture. The tube was placed in an ultrasonic bath for $6 \mathrm{~min}$, then the adsorption procedure was completed by vortexing for $1 \mathrm{~min}$ to accelerate the mass transfer of analytes through increasing the interfacial area between the solid sorbent and sample solution. The MTX-loaded GO (MTX/GO) centrifuged in 6,000 rpm for $1 \mathrm{~min}$ and the supernatant was filtered off. Afterward, $700 \mu$ of acetone as desorption solvent was added to the sedimented MTX/GO, this mixture sonicated for $3 \mathrm{~min}$, then filtered by centrifuge $6,000 \mathrm{rpm}$ for $1 \mathrm{~min}$. The supernatant was kept for analyzing the drug content. The acetone was evaporated by nitrogen gas, and the residue was dissolved in $200 \mu \mathrm{l}$ ACN. Finally, the amount of MTX was determined by UV spectrophotometer at $375 \mathrm{~nm}$.

\section{Results}

In order to improve the extraction capability, a set of effective parameters were studied including sample volume, $\mathrm{pH}$ of sample solution, amount of adsorbent, adsorption and desorption time and type of eluting solution. These preliminary studies were performed in human saliva samples. Extraction of MTX performed through $\pi-\pi$ interaction between aromatic part on drug structure and delocalized $\pi$-electron system of GO surface, also the formation of hydrogen bonding of carboxyl and amine group of MTX with carboxyl and hydroxyl groups on the surface of GO is considered.

\section{Sample volume}

For the analysis of a real sample using pre-concentration, the sample volume is one of the most important parameters for obtaining high enrichment factor. To do this, $0.5 \mathrm{ml}$ of the saliva sample after MTX spiking was used in all studied volumes. Volumes of sample solutions were changed from 1.0 to $10.0 \mathrm{ml}$ in test tubes. The highest analytical response was achieved at the sample volume of $3.0 \mathrm{ml}$. Therefore, $3.0 \mathrm{ml}$ was chosen as the optimum sample volume.

\section{pH optimization}

The $\mathrm{pH}$ values were investigated between 3.0 and 11.0 by the addition of $0.1 \mathrm{M} \mathrm{HCl}$ or $0.1 \mathrm{M} \mathrm{NaOH}$ solutions. From Figure 2A, it was revealed that the extraction has been occurred best in acidic condition. The MTX has three different $\mathrm{pKa}$ values, 3.8, 4.8 and 5.6 due to the different chemical sites. In $\mathrm{pH}=4$, the MTX is mostly in undissociated form, thus the solubility is reduced and there upon maximum extraction is reached. It can be concluded that the electrostatic interactions between MTX and adsorbent showed a moderate role in the adsorption process. Since the $\mathrm{pH}$ of the real saliva samples was generally in the range of 6.5-7.0, there it is needed to adjust the $\mathrm{pH}$ of the sample solution before the adsorption process.

\section{Amount of GO sorbent}

The optimum amount of the adsorbent for maximum take up was determined in the range of $2-5 \mathrm{mg} / \mathrm{ml}$. According to Figure 2B, $3 \mathrm{mg} / \mathrm{ml}$ of the adsobent was enough to pre-concentrate the MTX, at the studied concentrations.

\section{Adsorption time}

In this experiment, adsorption time means the time interval from the beginning of the injection and its end<smiles>CN(Cc1cnc2nc(N)nc(N)c2n1)c1ccc(C(=O)NC(CCC(=O)O)C(=O)O)cc1</smiles>

Figure 1. Chemical Structure of Methotrexate

Table 1. Assay Precision, Accuracy and Recovery of Saliva Samples Spiked with MTX

\begin{tabular}{|c|c|c|c|c|c|}
\hline Analyte & Nominal concentration $(\mu \mathrm{g} / \mathrm{ml})$ & Intra-day RSD $\% ; n=5^{a}$ & Inter-day RSD $\% ; n=5^{b}$ & Accuracy $(\mathrm{RE} \%)^{\mathrm{c}}$ & Recoveryd (\%) \\
\hline \multirow[t]{3}{*}{ MTX } & 25 & 5.44 & 7.04 & 4.54 & 104.54 \\
\hline & 50 & 7.05 & 6.19 & -2.15 & 97.85 \\
\hline & 100 & 3.45 & 3.50 & -0.04 & 99.96 \\
\hline
\end{tabular}

a , Number of replicates; ${ }^{b}$, Number of days; ${ }^{c}, \mathrm{RE} \%=100$; ((Found value-Nominal value)/ Nominal value). dRecovery $(\%)$ : Found value/Nominal concentration $\times 100$ 
Table 2. Evaluation of Method Stability of MTX in Saliva

\begin{tabular}{lccc}
\hline Analyte & Concentration added $(\mu \mathrm{g} / \mathrm{ml})$ & \multicolumn{2}{c}{ Recovery $(\% \mathrm{RR})$} \\
& & Freeze-thaw stability & Room temperature stability \\
\hline MTX & 50 & 104.46 & 104.27 \\
& 200 & 100.28 & 105.28 \\
& 700 & 98.25 & 94.22 \\
\hline
\end{tabular}

Table 3. Investigation of the Interference Effect on MTX Determination Obtained Under D-MSPESpectrophotometry in the Presence of $200 \mathrm{ng} / \mathrm{ml}$ MTX

\begin{tabular}{lc}
\hline Co-spiked drug with MTX & Accuracy (\%) \\
\hline Morphine & 7.8 \\
Vancomycin & 8 \\
Clonazepam & 7 \\
Cyclosporine & 0.5 \\
Citrizine & 0.5 \\
Atrovastatine & 1.1 \\
Acetaminophen & 3.2 \\
Diazepam & 7 \\
\hline
\end{tabular}

just before using an external magnetic field. The time of adsorption is a necessary parameter to ensure the sufficient contact between sorbent and analyte. In the present stage the effect of time on extraction efficiency was checked in different contact times; 2, 4, 6, 8 and $10 \mathrm{~min}$. As shown in Figure $2 \mathrm{C}$, the equilibrium of the extraction process was reached at 6 min so recovery increased in this time. Such a fast equilibrium between the adsorbent and analyte is reasonable due to the high hydrophilicity of adsorbent and its large accessible surface for extraction. With extending the time over 6 min, no significant change in analytical response was observed and subsequent experiments were set at $6 \mathrm{~min}$. This observation could be as the result of nanoparticle agglomeration upper than the equilibrium point then reduction of surface area for drug adsorption.

\section{Desorption conditions}

In order to choose the best eluent, MTX was desorbed with various eluting solutions such as $\mathrm{ACN}$, acetone, methanol, and ethanol. As shown in Figure 3A, the desorption capacity of acetone is much better than that

Table 4. Comparison of the Two Methods for the Analysis of MTX in the Saliva Samples of Patients Treated with MTX

\begin{tabular}{lcccc}
\hline Real sample \# & \multicolumn{2}{c}{ MTX level in saliva } & t-value & F-value \\
& ${\text { Given } \text { method }^{\mathrm{b}}}_{1}$ & Reference method & & \\
2 & $0.25 \pm 0.010 \mu \mathrm{g} / \mathrm{ml}$ & $0.19 \pm 0.003 \mu \mathrm{g} / \mathrm{ml}$ & 1.15 & 1.84 \\
\hline
\end{tabular}

${ }^{\mathrm{a}}$,The found values are average of three determinations \pm standard deviation; ${ }^{\mathrm{b}}$, Critical t-value and $\mathrm{F}$-value at $\% 95$ confidence level are 4.30 and 19.00. Experimental condition for reference method; Mobile phase, phosphate buffer $(\mathrm{pH}=3.6)$ :acetonitrile $(89: 11, \mathrm{v} / \mathrm{v})$; flow rate, $1 \mathrm{~mL} / \mathrm{min}$; $\lambda, 375 \mathrm{~nm}$.

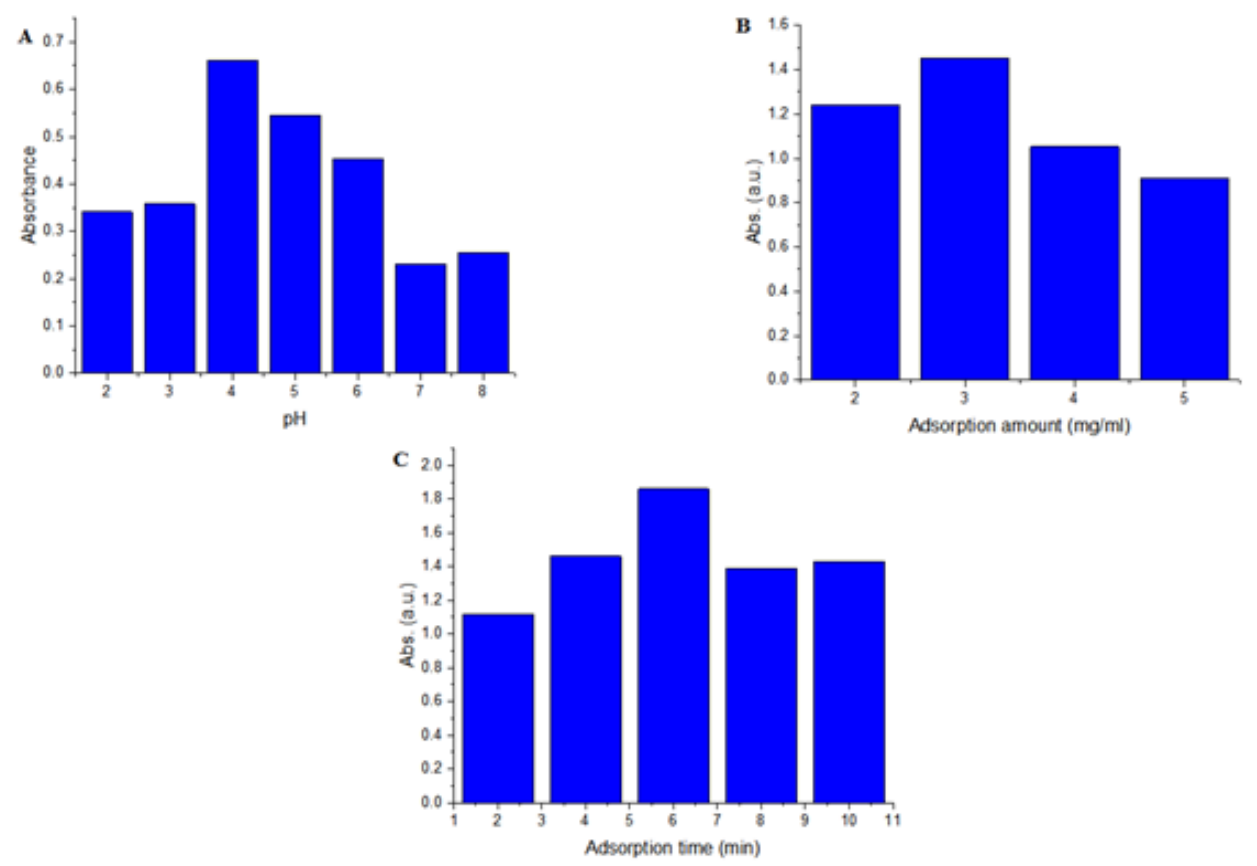

Figure 2. Effect of pH (A) Various Amounts of GO Sorbent (mg/mL) (B) and Contact Time (C) on the Extraction Efficiency 

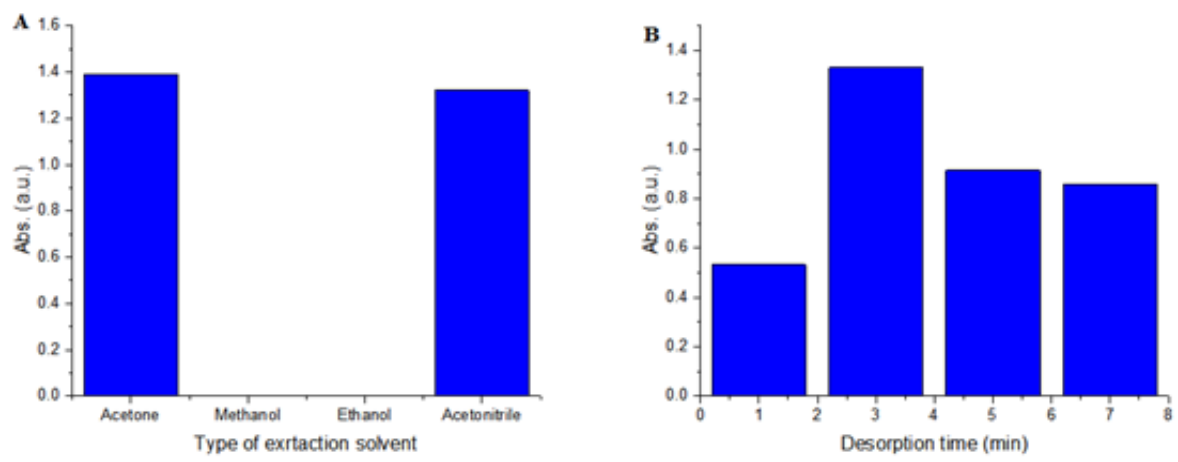

Figure 3. Effect of Type of Elution Solvent (A) and desorption solvent volume (B) on the extraction efficiency of the GO adsorbent.

other eluents. Acetone with highest extraction efficiency is selected as desorption solvent.

In order to complete desorption of the analyte from $\mathrm{GO}$, the effect of desorption time on desorption capacity was also evaluated. Desorption time (1, 3, 5, 7, and 9 min) was also investigated. As shown in Figure $3 \mathrm{~B}, 3 \mathrm{~min}$ is sufficient to desorb the MTX.

\section{Method validation}

The required parameters for validation of an analytical method according to the US FDA guideline are linearity, lower limit of quantification (LLOQ), upper limit of quantification (ULOQ), precision, accuracy, recovery, sensitivity, selectivity, and robustness (Food, 2001). In present paper required aspects for an analytical method have been covered including; linearity, accuracy, precision, recovery, stability, and selectivity.

\section{Linearity}

The calibration curve generated over the MTX concentration range of $0.01-1 \mu \mathrm{g} / \mathrm{ml}$, with three replicates at each concentration level. For spiked saliva samples the correlation coefficient was greater than 0.999. Linear regression analysis of the results yielded an equation of $\mathrm{y}=0.409$ CMTX $(\mu \mathrm{g} / \mathrm{ml})+0.142$. Figure 4 shows the calibration curve related spectrum obtained by spectrophotometer in an optimized condition.

\section{Precision, accuracy and recovery}

Precision was calculated in accordance with the FDA recommendation in two stages. Intra-day repeatability was assessed by analyzing five consecutive injections of MTX-spiked samples in three varying concentrations. The inter-day analysis was performed by quantification of the same concentrations on five consecutive days.

According to the FDA guideline the RSD\% values lower than $20 \%$ for LLOQ and $15 \%$ for other occasions which are acceptable for bioanalysis. The accuracy of the method was defined as a relative error (RE\%) calculated using the following equation:

$R E \%=100 \times\left(\frac{\text { Found value }- \text { Nominal value }}{\text { Nominal value }}\right) \quad$ Equation 1

The relative recovery ( $\mathrm{RR} \%$ ) is defined as the measured concentration of the drug divided by its actual concentration. The intra-day and inter-day precisions, accuracy and recovery were performed by five replicates of 25, 50, and $100 \mathrm{ng} / \mathrm{ml}$. The precision, accuracy, and recovery calculations are detailed in Table 1.

\section{Stability}

In this work, the stability tests covered the main concerns which target the stability of the components; when samples come to freezing as well as they come to stand at the bench. As the samples are severally frozen and thawed; thus, the effect of multi-freeze/thaw cycles should be concerned. The stability of the analyte should also be assessed in the sample preparation step until the analysis has been completed to figure out any degradation during the sample preparation. Table 2 shows the results of stability evaluation.
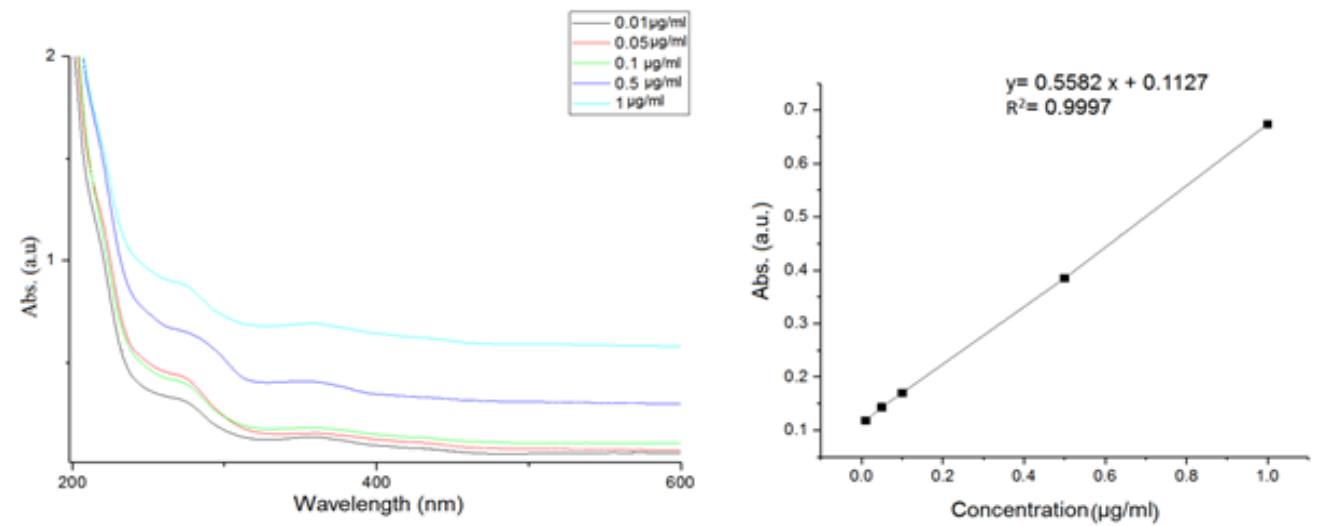

Figure 4. UV-vis Absorption Spectra of MTX at Increasing Known Concentrations 


\section{Selectivity}

For spectrophotometric determination, it is necessary to investigate the acceptable levels of selectivity. Sample preparation is the first step to prone the sensitive and selective analytical method. Selectivity demonstrates the ability of the developed method to quantify the intended analyte among the compounds present in the sample. According to the FDA guideline, the drug-free saliva sample was collected from three different sources and applied into the spectrophotometer after applying $D-\mu$-SPE. The blank samples showed no suspected interferences at absorbance area of the intended analyte and/or additional compounds did not have UV absorption at that wavelength. It means that routine constituents of extracted residual had no interferes with the analysis performance. So, the proposed method differentiates the target analyte in presence of potential interferences.

Selectivity of the method was ensured by adding $100 \mathrm{ng} / \mathrm{ml}$ of different probable co-administrated drugs (e.g., vancomycin, morphine, clonazepam, cetirizine, atorvastatin and cyclosporine) and most routinely administrated drugs such as acetaminophen and diazepam in saliva samples. The acceptable tolerance of accuracy should not exceeded $20 \%$ as was observed in the present study. Investigating of the interference effect of other drugs is presented in Table 3. As can be observed that the absorbance of investigated co-administrated drugs is not same as MTX. The obtained results confirmed that MTX could be successfully determined in real human saliva samples without the interference of a variety of routine compounds such as proteins, salts and usual coadministrated substances.

\section{Discussion}

\section{Application of D-MSPE procedure to real sample}

To evaluate the application of the present method, two saliva samples were taken from patients under MTX therapy and then analyzed by the optimum D- $\mu$-SPE condition. Table 4 shows the patient plasma MTX levels for both samples. The accuracy of the method was confirmed by HPLC measurements. Comparing the results obtained using the current and the reference methods by statistical analysis of the using Student's t-test and variance ratio F-test showed no statistically significant difference between the performance of the two methods regarding the accuracy and precision, respectively.

The performance of the newly developed $\mathrm{D}-\mu$-SPE/spectrophotometric method is comparable with other techniques from the viewpoints of a straightforward technique, LLOQ, apparatus, and validation tests (Albertioni et al., 1995; Hirai et al., 1997; Emara et al., 1998; Turci et al., 2000; Kuo et al., 2006; Chen and Zhang, 2008; Moghbel et al., 2010; Jouyban et al., 2011; Begas et al., 2013; Rodin et al., 2013; Wang et al., 2014; Wei et al., 2014; Wu et al., 2015; Fabrizi et al., 2016; Asadian et al., 2017). For a number of MTX determination LC methods cited in other reports, sophisticated detection systems such as MS or fluorescence were used which are not available in routine bioanalytical laboratories. The present method provided comparable LLOQ $(0.01 \mu \mathrm{g} / \mathrm{ml})$ in comparison with such sophisticated detectors. In addition, the sample preparation step in most of the methods are performed by tedious and time-consuming SPE or LLE techniques. The Present D- $\mu$-SPE method is an improved form of extraction in terms of sensitivity, rapidity, and easiness facility to fix the limitation of both LLE and SPE methods to a great extent.

In conclusion, a comparison of the proposed D- $\mu-\mathrm{SPE}$ method with other methods shows that D- $\mu$-SPE is a competitive spectrophotometric approach for MTX determination. The consumption of organic solvents in this method is much lower than that in LLE method. The results demonstrate the effectiveness of the $\mathrm{D}-\mu$-SPE in quantitatively extracting and pre-treating MTX without tedious pre-treatment procedure and expensive instrumentation. The graphene-based adsorbent was easily prepared and can be well dispersed in the saliva matrix separated from the medium by a centrifuge. This technique is very fast, because the extraction and desorption process take less time (less than $10 \mathrm{~min}$ ). $\mathrm{D}-\mu$-SPE is very simple, low cost, and suitable for clinical operations, which could greatly shorten the sample treatment time. This method is precise and accurate with low quantification limit. The validation criteria meet the FDA requirements very well. This simple method can be applied for the determination of MTX in patients saliva in clinics and laboratories.

\section{References}

Albertioni F, Pettersson B, Beck O, et al (1995). Simultaneous quantitation of methotrexate and its two main metabolites in biological fluids by a novel solid-phase extraction procedure using high-performance liquid chromatography. J Chromatogr B, 665, 163-70.

Asadian E, Shahrokhian S, Zad AI, et al (2017). Glassy carbon electrode modified with 3D graphene-carbon nanotube network for sensitive electrochemical determination of methotrexate. Sensor Actuat B-Chem, 239, 617-27.

Ashri NY, Abdel-Rehim M (2011). Sample treatment based on extraction techniques in biological matrices. Bioanalysis, 3, 2003-18.

Ayazi Z (2017). Application of nanocomposite-based sorbents in microextraction techniques: a review. Analyst, 142, 721-39.

Begas E, Papandreou C, Tsakalof A, et al (2013). Simple and reliable HPLC method for the monitoring of methotrexate in osteosarcoma patients. J Chromatogr Sci, 52, 590-5.

Chen S, Zhang Z (2008). Molecularly imprinted solid-phase extraction combined with electrochemical oxidation fluorimetry for the determination of methotrexate in human serum and urine. Spectrochim Acta A-M, 70, 36-41.

Emara S, Askal H, Masujima T (1998). Rapid determination of methotrexate in plasma by high-performance liquid chromatography with on-line solid-phase extraction and automated precolumn derivatization. Biomed Chromatogr, 12, 338-42.

Fabrizi G, Fioretti M, Mainero Rocca L (2016). Dispersive solid-phase extraction procedure coupled to UPLC-ESI-MS/ MS analysis for the simultaneous determination of thirteen cytotoxic drugs in human urine. Biomed Chromatogr, 30, 1297-308.

Fleisher M (1993). Antifolate analogs: mechanism of action, analytical methodology, and clinical efficacy. Ther Drug Monit, 15, 521-6. 
Flores JR, Peñalvo GC, Mansilla AE, et al (2005). Capillary electrophoretic determination of methotrexate, leucovorin and folic acid in human urine. J Chromatogr B, 819, 141-7.

Food U (2001). Drug Administration FDA Guidance for Industry: Bioanalytical Method Validation. US Department of Health and Human, Services Food and Drug Administration. and Center for Drug Evaluation and Research.

Gallardo E, Queiroz J (2008). The role of alternative specimens in toxicological analysis. Biomed Chromatogr, 22, 795-821.

Ghasemi J, Niazi A (2005). Two-and three-way chemometrics methods applied for spectrophotometric determination of lorazepam in pharmaceutical formulations and biological fluids. Anal Chim Acta, 533, 169-77.

Hamidi S, Alipour-Ghorbani N (2017). Liquid-phase microextraction of biomarkers: A review on current methods. $J$ Liq Chromatogr R T, 40, 853-61.

Hamidi S, Alipour-Ghorbani N, Hamidi A (2018). Solid phase microextraction techniques in determination of biomarkers. Crit Rev Anal Chem, 48, 239-51.

Hamidi S, Amini M, Khoubnasabjafari M, et al (2017). LC-MS/MS estimation of propranolol level in exhaled breath condensate. Pharm Sci, 23, 264-70.

Hamidi S, Jouyban A (2015). Capillary electrophoresis with UV detection, on-line stacking and off-line dispersive liquid-liquid microextraction for determination of verapamil enantiomers in plasma. Anal Methods, 7, 5820-9.

Hamidi S, Jouyban A (2015). Pre-concentration approaches combined with capillary electrophoresis in bioanalysis of chiral cardiovascular drugs. Pharm Sci, 21, 229-43.

Hamidi S, Khoubnasabjafari M, Ansarin K, et al (2016). Direct analysis of methadone in exhaled breath condensate by capillary zone electrophoresis. Curr Pharm Anal, 12, 137-45.

Hamidi S, Khoubnasabjafari M, Ansarin K, et al (2017). Chiral separation of methadone in exhaled breath condensate using capillary electrophoresis. Anal Methods, 9, 2342-50.

Hamidi S, Soltani S, Jouyban A (2015). A dispersive liquid-liquid microextraction and chiral separation of carvedilol in human plasma using capillary electrophoresis. Bioanalysis, 7, 1107-17.

Hirai T, Matsumoto S, Kishi I (1997). Determination of methotrexate and its main metabolite 7-hydroxymethotrexate in human urine by high-performance liquid chromatography with normal solid-phase extraction. J Chromatogr B, 690, 267-73.

Hroch M, Tuková J, Doležalová P, et al (2009). An improved high-performance liquid chromatography method for quantification of methotrexate polyglutamates in red blood cells of children with juvenile idiopathic arthritis. Biopharm Drug Ddispos, 30, 138-48.

Jafari S, Hamidi S (2018). Microextraction techniques in antibiotic monitoring in body fluids: Recent trends and future. J Liq Chromatogr R T, 41, 401-7.

Jouyban A, Hamidi S (2017). Dispersive micro solid phase extraction using carbon-based adsorbents for sensitive determination of verapamil in plasma samples coupled with CE J Sep Sci, 16, 3318-26.

Jouyban A, Khoubnasabjafari M, Ansarin K, et al (2017). Methadone concentrations in exhaled breath condensate, serum and urine of patients under maintenance treatment. Iranian J Pharm Res, 16, 1621.

Jouyban A, Shaghaghi M, Manzoori JL, et al (2011). Determination of methotrexate in biological fluids and a parenteral injection using terbium-sensitized method. Iranian J Pharm Res, 10, 695.

Khezeli T, Daneshfar A (2017). Development of dispersive micro-solid phase extraction based on micro and nano sorbents. TrAC Trend Anal Chem, 89, 99-118.
Kuo CY, Chiou SS, Wu SM (2006). Solid-phase extraction and large-volume sample stacking with an electroosmotic flow pump in capillary electrophoresis for determination of methotrexate and its metabolites in human plasma. Electrophoresis, 27, 2905-9.

Li Yd, Li Y, Liang Ns, et al (2015). A reversed-phase high performance liquid chromatography method for quantification of methotrexate in cancer patients serum. J Chromatogr B, 1002, 107-12.

Moghbel A, Zand-Moghaddamb A, Rezaee S, et al (2010). High-performance liquid chromatography determination of methotrexate in plasma. Iranian J Pharm Res, 10, 149-52.

Nelson JA, Harris BA, Decker WJ, et al (1977). Analysis of methotrexate in human plasma by high-pressure liquid chromatography with fluorescence detection. Cancer Res, 37, 3970-3.

Poole CF (2003). New trends in solid-phase extraction. TrAC Trend Anal Chem, 22, 362-73.

Rodin I, Braun A, Stavrianidi A, et al (2013). A validated LCMS/MS method for rapid determination of methotrexate in human saliva and its application to an excretion evaluation study. J Chromatogr B, 937, 1-6.

Rubino FM (2001). Separation methods for methotrexate, its structural analogues and metabolites. $J$ Chromatogr B, 764, 217-54.

Ruiz-Gutiérrez V, Pérez-Camino MdC (2000). Update on solid-phase extraction for the analysis of lipid classes and related compounds. J Chromatogr A, 885, 321-41.

Samari F, Hemmateenejad B, Shamsipur M (2010). Spectrophotometric determination of carminic acid in human plasma and fruit juices by second order calibration of the absorbance spectra- $\mathrm{pH}$ data matrices coupled with standard addition method. Anal Chim Acta, 667, 49-56.

Shahriary L, Athawale AA (2014). Graphene oxide synthesized by using modified hummers approach. Int J Renew Energy Environ Eng, 2, 58-63.

Turci R, Fiorentino ML, Sottani C, et al (2000). Determination of methotrexate in human urine at trace levels by solid phase extraction and high-performance liquid chromatography/ tandem mass spectrometry. Rapid Commun Mass Sp, 14, 173-9.

Uchiyama M, Matsumoto T, Matsumoto T, et al (2012). Simple and sensitive HPLC method for the fluorometric determination of methotrexate and its major metabolites in human plasma by post-column photochemical reaction. Biomed Chromatogr, 26, 76-80.

Vas G, Vekey K (2004). Solid-phase microextraction: a powerful sample preparation tool prior to mass spectrometric analysis. J Mass Spectrom, 39, 233-54.

Wang Y, Xie J, Tao L, et al (2014). Simultaneous electrochemical determination of epirubicin and methotrexate in human blood using a disposable electrode modified with nano-Au/ MWNTs-ZnO composites. Sensor Actuat B-Chem, 204, 360-7.

Wei Y, Luo L, Ding Y, et al (2014). Highly sensitive determination of methotrexate at poly (1-lysine) modified electrode in the presence of sodium dodecyl benzene sulfonate. Bioelectrochemistry, 98, 70-5.

Wu D, Wang Y, Sun Y, et al (2015). A simple, rapid and reliable liquid chromatography-mass spectrometry method for determination of methotrexate in human plasma and its application to therapeutic drug monitoring. Biomed Chromatogr, 29, 1197-202.

Yang J, Tan X, Shih W-C, et al (2014). A sandwich substrate for ultrasensitive and label-free SERS spectroscopic detection of folic acid/methotrexate. Biomed Microdevices, 16, 673-9.

Ye N, Shi P (2015). Applications of graphene-based materials 
Fatemeh Soghra Jahed et al

in solid-phase extraction and solid-phase microextraction. Sep Purif Rev, 44, 183-98.

\section{(0) $(0)$}

This work is licensed under a Creative Commons AttributionNon Commercial 4.0 International License. 